\title{
MECI: A Method for Eclipsing Component Identification
}

\section{Citation}

Devor, Jonathan, and David Charbonneau. 2006. “MECI: A Method for Eclipsing Component Identification." The Astrophysical Journal 653 (1): 647-56. https://doi.org/10.1086/508609.

\section{Permanent link}

http://nrs.harvard.edu/urn-3:HUL.InstRepos:41397439

\section{Terms of Use}

This article was downloaded from Harvard University's DASH repository, and is made available under the terms and conditions applicable to Other Posted Material, as set forth at http:// nrs.harvard.edu/urn-3:HUL.InstRepos:dash.current.terms-of-use\#LAA

\section{Share Your Story}

The Harvard community has made this article openly available.

Please share how this access benefits you. Submit a story.

\section{Accessibility}


The Astrophysical Journal, 653:647-656, 2006 December 10

(C) 2006. The American Astronomical Society. All rights reserved. Printed in U.S.A.

\title{
MECI: A METHOD FOR ECLIPSING COMPONENT IDENTIFICATION
}

\author{
Jonathan Devor and David Charbonneau ${ }^{1}$ \\ Harvard-Smithsonian Center for Astrophysics, 60 Garden Street, Cambridge, MA 02138; jdevor@cfa.harvard.edu \\ Received 2006 July 23; accepted 2006 August 21
}

\begin{abstract}
We describe an automated method for assigning the most probable physical parameters to the components of an eclipsing binary, using only its photometric light curve and combined colors. With traditional methods, one attempts to optimize a multiparameter model over many iterations, so as to minimize the $\chi^{2}$ value. We suggest an alternative method, in which one selects pairs of coeval stars from a set of theoretical stellar models and compares their simulated light curves and combined colors with the observations. This approach greatly reduces the parameter space over which one needs to search and allows one to estimate the components' masses, radii, and absolute magnitudes, without spectroscopic data. We have implemented this method in an automated program using published theoretical isochrones and limb-darkening coefficients. Since it is easy to automate, this method lends itself to systematic analyses of data sets consisting of photometric time series of large numbers of stars, such as those produced by OGLE, MACHO, TrES, HAT, and many others surveys.
\end{abstract}

Subject headings: binaries: eclipsing — methods: data analysis — stars: statistics — techniques: photometric

\section{INTRODUCTION}

Eclipsing double-lined spectroscopic binaries provide the only method by which both the masses and radii of stars can be estimated without having to resolve the binary spatially or rely on astrophysical assumptions. Despite the large variety of models and parameter-fitting implementations (e.g., WD [Wilson \& Devinney 1971] and EBOP [Etzel 1981; Popper \& Etzel 1981]), their underlying methodology is essentially the same. Photometric data provide the light curve of the eclipsing binary (EB), and spectroscopic data provide the radial velocities of its components. The depth and shape of the light-curve eclipses constrain the components' brightness and fractional radii, while the radial velocity sets the length scale of the system. In order to fully characterize the components of the binary, one needs to combine all of this information. Only a small fraction of all binaries eclipse, and spectra with sufficient resolution and signal-to-noise ratios can be gathered only for bright stars. The intersection of these two groups leaves a small number of stars.

Over the past decade, the number of stars with high-quality, multiepoch, photometric data has grown dramatically due to the growing interest in finding gravitational lensing events (Wambsganss 2006) and eclipsing extrasolar planets (Charbonneau et al. 2006). In addition, major technical improvements in both CCD detectors and implementations of image-difference analysis techniques (Crotts 1992; Alard \& Lupton 1998; Alard 2000) enable simultaneous photometric measurements of tens of thousands of stars in a single exposure. Today, there are many millions of light curves available from a variety of surveys, such as OGLE (Udalski et al. 1994), MACHO (Alcock et al. 1998), TrES (Alonso et al. 2004; O'Donovan 2006), HAT (Bakos et al. 2004), and XO (McCullough et al. 2006). Despite the increase in photometric data, there has not been a corresponding growth in the quantity of spectroscopic data, nor is this growth likely to occur in the near future. Thus, the number of fully characterized EBs has not grown at a rate commensurate with the available photometric data sets.

\footnotetext{
${ }^{1}$ Alfred P. Sloan Research Fellow.
}

In recent years, there has been a growing effort to mine the wealth of available photometric data by employing automated pipelines that use simplified EB models in the absence of spectroscopic observations and hence without a fixed physical length scale or absolute luminosity (Wyithe \& Wilson 2001, 2002; Devor 2004, 2005). In this paper, we present a method that utilizes theoretical isochrones and multiepoch photometric observations of the binary system to estimate the physical parameters of the component stars, while still not requiring spectroscopic observations.

Our Method for Eclipsing Component Identification (MECI) ${ }^{2}$ finds the most probable masses, radii, and absolute magnitudes of the stars. The input for MECI is an EB's photometric light curve and out-of-eclipse colors (we note that in the absence of color information, the accuracy in the estimation of the stellar parameters is significantly reduced, see $\S 4.2$ ). This approach can be used to quickly characterize large numbers of eclipsing binaries; however, it is not sufficient to improve stellar models, since underlying isochrones must be assumed.

In a previous paper (Devor \& Charbonneau 2006), we outlined the ideas behind both MECI and a closely related, "quick and dirty" alternative, which we called "MECI-express." Although MECI-express is much faster and easier to implement, it is also far less accurate. For this reason we will not discuss it further and instead concentrate exclusively on MECI. We discuss its applications in $\S 2$, aspects of its implementation in $\S 3$, and tests of its accuracy in $\S 4$, and, finally, we summarize our findings in $\S 5$.

\section{MOTIVATION}

\subsection{Characterizing the Binary Stellar Population}

First and foremost, MECI is designed as a high-throughput means to systematically estimate the masses of large numbers of stars. Although the result in each system is uncertain, by

\footnotetext{
2 The source code and running examples of MECI, as well as a suite of utilities, can be downloaded from: http://cfa-www.harvard.edu/ jdevor/MECI.html.
} 
statistically analyzing large catalogs, one can reduce the nonsystematic errors. Much work has already been invested into characterizing binary systems through spectroscopic binary surveys (e.g., Duquennoy \& Mayor 1991; Pourbaix et al. 2004), yet the limited data and their large uncertainties have led to inconsistent results (Mazeh et al. 2006). The driving questions that have spurred debate in the community include: What are the initial mass functions of the primary and secondary components? How do they relate to the initial mass function of single stars? What is the distribution of the components' mass ratio, $q$, and in particular, does it peak at unity? This lack of understanding is further highlighted by the fact that most of the stars in our galaxy are members of binary systems, and that these questions have lingered for over a century. MECI may help sort this out by systematically characterizing the component stars of many EB systems.

By requiring only photometric data, a survey using MECI can study considerably fainter binary systems than spectroscopic surveys and thus remain complete to a far larger volume. As an illustrative example, the difference image analysis of the bulge fields of OGLE II, using the Las Campanas $1.3 \mathrm{~m}$ Warsaw telescope in a drift-scan mode (an effective exposure time of $87 \mathrm{~s}$ ), attained a median noise level of $0.1 \mathrm{mag}$ for $I=18$ binaries, even in moderately crowded fields (Wozniak 2000). In contrast to this, the CfA digital speedometer on the $1.5 \mathrm{~m} \mathrm{~F}$. L. Whipple Observatory telescope has a spectral resolution of $R \simeq 35,000$ (at 5177 $\AA$ ) and typically yields a radial velocity precision of $0.5 \mathrm{~km} \mathrm{~s}^{-1}$, with a faint magnitude limit of $V=13$ (Latham 1992). Although the limiting magnitudes are very much dependent on the throughput of the relevant instruments and the precision one wishes to achieve, this 5 mag difference for telescopes of similar aperture corresponds to a factor of 10 in distance or 1000 in volume, and illustrates the significant expansion that can be achieved by purely photometric surveys. Conversely, one can achieve the same magnitude limit with an aperture 10 times smaller. The success of this approach has been demonstrated by several automated observatories, such as TrES (Alonso et al. 2004) and HAT (Bakos et al. 2004), which each use networks of observatories with $10 \mathrm{~cm}$ camera lenses to monitor stars to $V \simeq 13$.

\subsection{Identifying Low-Mass Main-Sequence EBs}

One of the most compelling applications of MECI will be to quickly sort thousands of EBs present in large photometric surveys and subsequently select a small subset of objects from the resulting catalog for further study. In particular, lower mainsequence stars that are partially or fully convective have not been studied with a level of detail remotely approaching that of solartype (and more massive) stars. This is particularly troubling since late-type stars are the most common in the Galaxy and dominate its stellar mass. It has been shown that models underestimate the radii of low-mass stars by as much as $20 \%$ (Lacy 1977a; Torres \& Ribas 2002); a significant discrepancy considering that for solar-type stars the agreement with the observations is typically within 1\%-2\% (Andersen 1998). Similar problems exist for the effective temperatures predicted theoretically for low-mass stars. Progress in this area has been hampered by the lack of suitable M dwarf binary systems with accurately determined stellar properties, such as mass, radius, luminosity, and surface temperature. Detached eclipsing systems are ideal for this purpose, but only five are known among M-type stars: CM Dra (Lacy 1977b; Metcalfe et al. 1996), YY Gem (Kron 1952; Torres \& Ribas 2002), CU Cnc (Delfosse et al. 1999; Ribas 2003), OGLE BW3 V38 (Maceroni \& Rucinski 1997; Maceroni \& Montalbán 2004), and
TrES-Her0-07621 (Creevey et al. 2005). They range in mass from about $0.25 \mathcal{M}_{\odot}\left(\mathrm{CM}\right.$ Dra) to $0.6 \mathcal{M}_{\odot}(\mathrm{YY} \mathrm{Gem})$. The number of such objects could be greatly increased by using tools such as MECI to mine the extant photometric data sets and locate these elusive low-mass systems.

\subsection{EBs as Standard Candles}

Using MECI, we are able to estimate the absolute magnitude of the binary system. This, together with its extinction-corrected out-of-eclipse apparent magnitude, allows us to then calculate the distance modulus to any given EB. The estimation of distances to EBs dates back to Stebbing (1910), and their use as distance candles in the modern astrophysical context was recently elucidated by Paczynski (1997). However, unlike these studies, MECI does not require spectroscopy and therefore is able to analyze binaries that are significantly less luminous (see $\S 2.1$ ). Although the distance estimation from MECI will be uncertain, in many cases this will still be an improvement over existing methods. For example, if there are many EBs in a stellar cluster, the distance estimate can be greatly improved by combining their results, reducing the nonsystematic errors by a factor of the square root of the number of systems. Following Guinan et al. (1996) one might be able to use such clustered EB standard candles to better constrain the distance to the LMC and SMC and thus be able to further constrain the bottom of the cosmological distance ladder. In the case of MECI, the uncertainties of each distance measurement will be considerably larger, but as suggested by T. Mazeh (2005, private communication), this will be compensated for by the far larger number of measurements that can be made. Another intriguing application of such EB standard candles is to map large-scale structures in the Galaxy, such as the location and orientation of the Galactic bar, arms, and merger remnants (see, e.g., Vallée 2005 and references therein).

\section{METHOD}

The EB component identification is performed in two stages. First the orbital parameters of the EB are estimated ( $\$ 3.1)$, then the most likely stellar parameters are identified (§ 3.2). Our implementation of MECI has the option to fix the estimates of the orbital parameters or to fine-tune them for each stellar pairing considered in the second stage. The average running time for MECI to analyze a 1000 point light curve on a single $3.4 \mathrm{GHz}$ Intel Xeon CPU is 0.4 minutes. If we permit fine-tuning of the orbital parameters for each pairing, the running time grows to 6 minutes per light curve.

\subsection{Stage 1: Finding the Orbital Parameters}

In the first stage, we estimate the EB's orbital parameters from its light curve. Many EBs have orbital periods of a few days or less, owing to the greater probability for such systems to present mutual eclipses and to the limited baselines in the data sets from which they are identified. Most of these systems will have orbits that have been circularized due to tidal effects. For such circular orbits, the only parameters we seek are the orbital period, $P$, and epoch of periastron, $t_{0}$. For noncircular orbits, we also fit the orbital eccentricity, $e$, and the argument of periastron, $\omega$. The period is determined using a periodogram, and the remaining parameters are obtained through fitting the offset, duration, and time interval between the light curve's eclipses (see below). Holding these parameters fixed at these initial estimates significantly reduces the computational requirements of MECI. 

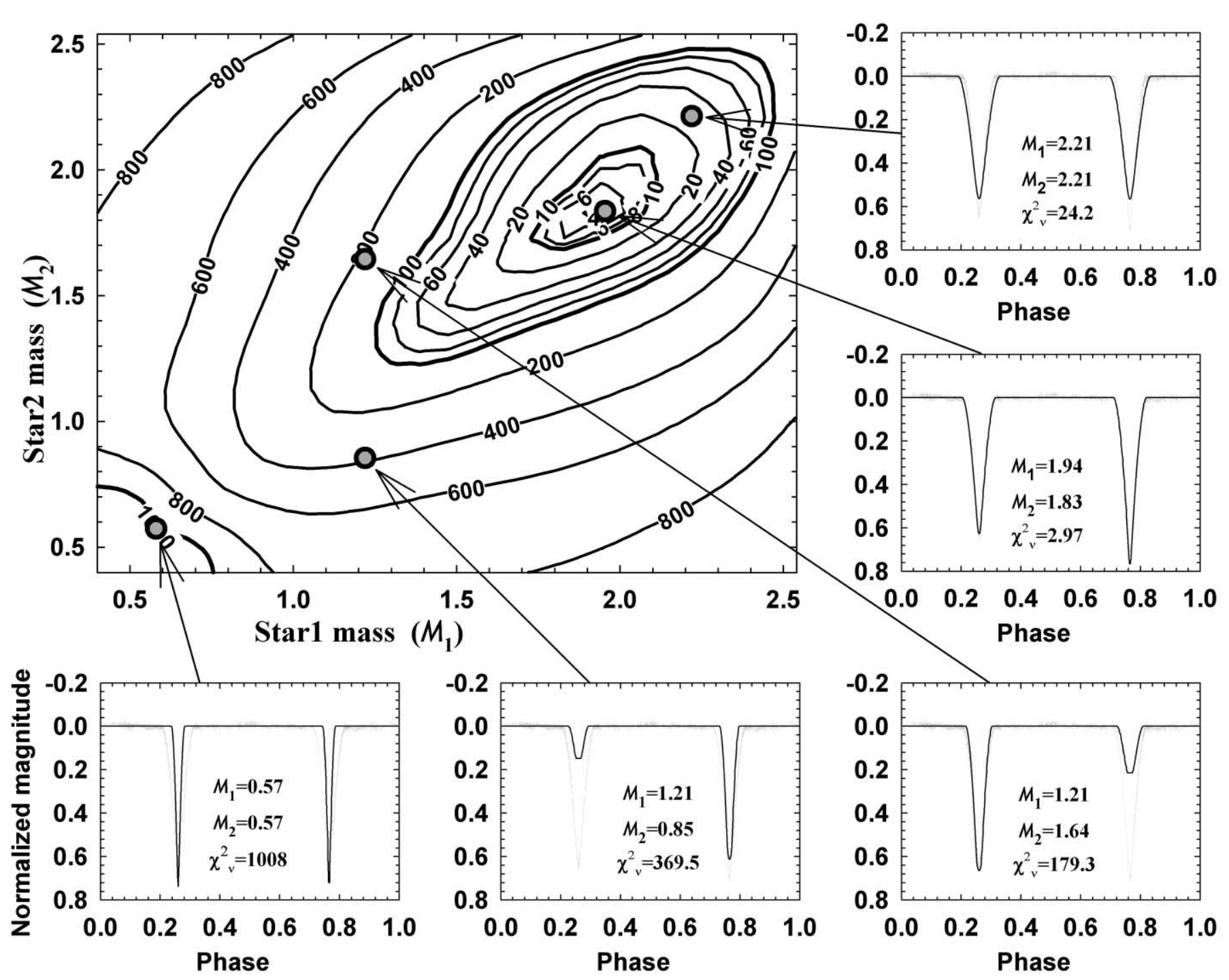

FIG. 1.-Upper-left panel: MECI $\chi_{\nu}^{2}$ surface as a function of the assumed masses (in units of $\mathcal{M}_{\odot}$ ) of the component stars in the WW Camelopardalis system. The model light curve at five locations in the grid is shown in the smaller panels, overplotted on the observed light curve from Lacy et al. (2002).

We postpone fitting the orbital inclination, $i$, until the second stage, since it is difficult to determine this parameter robustly without first assuming values for the stellar radii and masses. This difficulty arises because it is often difficult to distinguish a small secondary component from a large secondary component in a grazing orbit. In stage 2 , additional information, such as the theoretical stellar mass-radius relation and colors, are used to help resolve this degeneracy.

The procedure for fitting the aforementioned parameters from the EB light curve is a well-studied problem (Kopal 1959; Wilson \& Devinney 1971; Etzel 1991). We chose to estimate the period with a variant of the analysis of variances (AOV) periodogram ${ }^{3}$ by Schwarzenberg-Czerny $(1989,1996)$. We then use the Detached Eclipsing Binary Light curve (DEBiL) fitter ${ }^{4}$ by Devor (2004, 2005) for fitting the remaining orbital parameters. For noncircular systems, following Kopal (1959) and Kallrath \& Milone (1999), we estimate the orbital eccentricity and argument of periastron

\footnotetext{
3 The source code and running examples of both the AOV periodogram and the DEBiL fitter can be downloaded from: http://cfa-www.harvard.edu/ jdevor/ DEBiL.html.

${ }^{4}$ See footnote 3.
}

from the orbital period, the duration of the eclipses, $\Theta_{1,2}$, and the time interval between the eclipse centers, $\Delta t$, as

$$
\begin{aligned}
& \omega \simeq \arctan \left[\frac{2}{\pi}\left(\frac{\Theta_{1}-\Theta_{2}}{\Theta_{1}+\Theta_{2}}\right)\left(\frac{\Delta t}{P}-\frac{1}{2}\right)^{-1}\right], \\
& e \simeq \frac{\pi}{2 \cos \omega}\left|\frac{\Delta t}{P}-\frac{1}{2}\right| .
\end{aligned}
$$

In practice, it is difficult to accurately determine the eclipse duration. We estimate this duration by first calculating the median flux outside the eclipses, then estimating the midpoints and depths of the eclipses using a spline. We then assign the duration of each eclipse to be the time elapsed from the moment at which the light curve during ingress crosses the midpoint between the outof-eclipse and bottom-of-eclipse fluxes until the moment at which the light curve crosses the corresponding point during egress.

\subsection{Stage 2: Finding the Absolute Stellar Parameters}

In the second stage, we estimate the EB's absolute stellar parameters by iterating through many possible stellar pairings, 


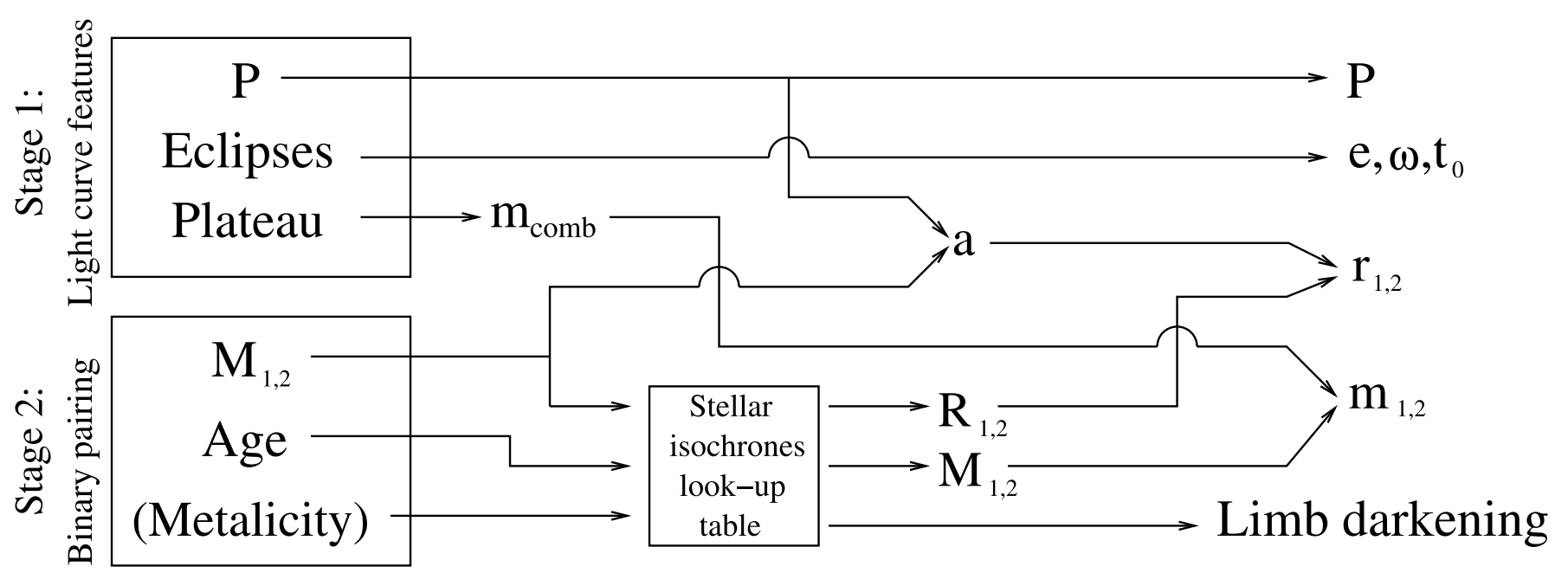

FIG. 2.-Flow diagram demonstrating the process by which MECI assigns the parameters to an EB based on its observed light curve. The details of stages 1 and 2 are described in $\S 3.1$ and $\S 3.2$, respectively.

simulating their expected light curves (see Fig. 1), and finding the pairing that minimizes the $\chi_{\nu}^{2}$ function (see $\S 3.3$ ). The parameters we fit are the masses of the two EB components, $\mathcal{M}_{1,2}$, their age (the components are assumed to be coeval), and their orbital inclination, $i$. Optionally, we can also fine-tune the orbital parameters obtained from the first stage. This option is necessary only for binaries with eccentric orbits, since varying their inclination will affect the fit of their previously estimated orbital parameters. The flow diagram for the entire procedure is shown in Figure 2.

If an estimate of the out-of-eclipse combined apparent magnitude, $m_{\text {comb }}$, of the EB (i.e., the light-curve plateau) is available, we may also estimate the distance modulus. If $m_{\text {comb }}$ is not available (e.g., if the light curve has been normalized), the distance modulus cannot be evaluated unless an independent measurement of the out-of-eclipse brightness is available. In either case, this procedure does not affect our estimates of the stellar parameters.

Once we assume the masses and age of the binary components, we use precalculated theoretical tables to look up their absolute stellar parameters, namely their radii, $R_{1,2}$, and absolute magnitudes, $M_{1,2}$. We use the Yonsei-Yale isochrones of solar metallicity (Kim et al. 2002) to specify the binary components' radii and absolute magnitudes, in a range of filters $(U, B, V, R, I)_{\mathrm{C}}$ and $(J H K)_{\mathrm{ESO}}$. We note that the Yonsei-Yale isochrones do not extend below $0.4 \mathcal{M}_{\odot}$. To consider stars with masses below this value, we constructed tables from the isochrones of Baraffe et al. (1998), which are generally more reliable for masses below $0.75 \mathcal{M}_{\odot}$.

Together with the orbital parameters $(\S 3.1)$, we have all the information required to simulate the EB light curve. The fractional radii, $r_{1,2}$, and apparent magnitudes, $m_{1,2}$, of the binary components, which are needed for this calculation, are calculated as

$$
\begin{aligned}
a & =\left[G\left(\mathcal{M}_{1}+\mathcal{M}_{2}\right)(P / 2 \pi)^{2}\right]^{1 / 3} \\
& \simeq 4.206 R_{\odot}\left(\mathcal{M}_{1} / \mathcal{M}_{\odot}+\mathcal{M}_{2} / \mathcal{M}_{\odot}\right)^{1 / 3}(P / \text { day })^{2 / 3}, \\
r_{1,2} & =R_{1,2} / a \\
m_{1} & =m_{\mathrm{comb}}+2.5 \log \left(1+10^{-0.4\left(M_{2}-M_{1}\right)}\right), \\
m_{2} & =m_{1}+\left(M_{2}-M_{1}\right) .
\end{aligned}
$$

We create model light curves using DEBiL, which has a fast light-curve generator. DEBiL assumes that the EB is detached, with limb-darkened spherical components (i.e., no tidal distortions or reflections). To describe the stellar limb darkening, it employs the quadratic law (Claret et al. 1995):

$$
I(\theta)=I_{0}\left[1-\tilde{a}(1-\cos \theta)-\tilde{b}(1-\cos \theta)^{2}\right]
$$

where $\theta$ is the angle between the line of sight and the emergent flux, $I_{0}$ is the flux at the center of the stellar disk, and $\tilde{a}$ and $\tilde{b}$ are coefficients that define the amplitude of the center-to-limb variations. We use the ATLAS (Kurucz 1992) and PHOENIX (Claret $1998,2000)$ tables to look up the quadratic limb-darkening coefficients for high-mass $\left(T_{\text {eff }} \geq 10000 \mathrm{~K}\right.$ or $\left.\log g \leq 3.5\right)$ and lowmass $\left(T_{\text {eff }}<10000 \mathrm{~K}\right.$ and $\left.\log g>3.5\right)$ main-sequence stars, respectively.

Finally, the orbital inclination is fit at each iteration so to make the simulated light curve most similar to the observations. For this we employed the robust "golden section" bracket search algorithm (Press et al. 1992). This inner loop dominates the computational time required. In the case of noncircular orbits, it is often necessary to iterate the estimates of the orbital parameters $\left(e, t_{0}\right.$, $\omega$, and $i$ ). When this option is enabled, MECI employs the rolling simplex algorithm (Nelder \& Mead 1965; Press et al. 1992), which fits all four orbital parameters simultaneously.

\subsection{Assessing the Likelihood of a Binary Pairing}

The observational data for each EB consists of $N_{\text {lc }}$ observed magnitudes $O_{i}$, each with an associated uncertainty $\epsilon_{i}$, as well as $N_{\text {colors }}$ out-of-eclipse colors $\tilde{O}_{c}$, each with an uncertainty $\tilde{\epsilon}_{c}$. Our model yields the corresponding predicted light-curve magnitudes $C_{i}$ and out-of-eclipse colors $\tilde{C}_{c}$. We define the goodness-of-fit function to be

$$
\chi_{\nu}^{2}=\frac{1}{w+N_{\text {colors }}}\left[\frac{w}{N_{\mathrm{lc}}} \sum_{i=1}^{N_{\mathrm{lc}}}\left(\frac{O_{i}-C_{i}}{\epsilon_{i}}\right)^{2}+\sum_{c=1}^{N_{\text {colors }}}\left(\frac{\tilde{O}_{c}-\tilde{C}_{c}}{\tilde{\epsilon}_{c}}\right)^{2}\right],
$$

where $w$ is a factor that describes the relative weights assigned to the light curve and color data (see below). The value of $\chi_{\nu}^{2}$ should achieve unity if the assumed model accurately describes 


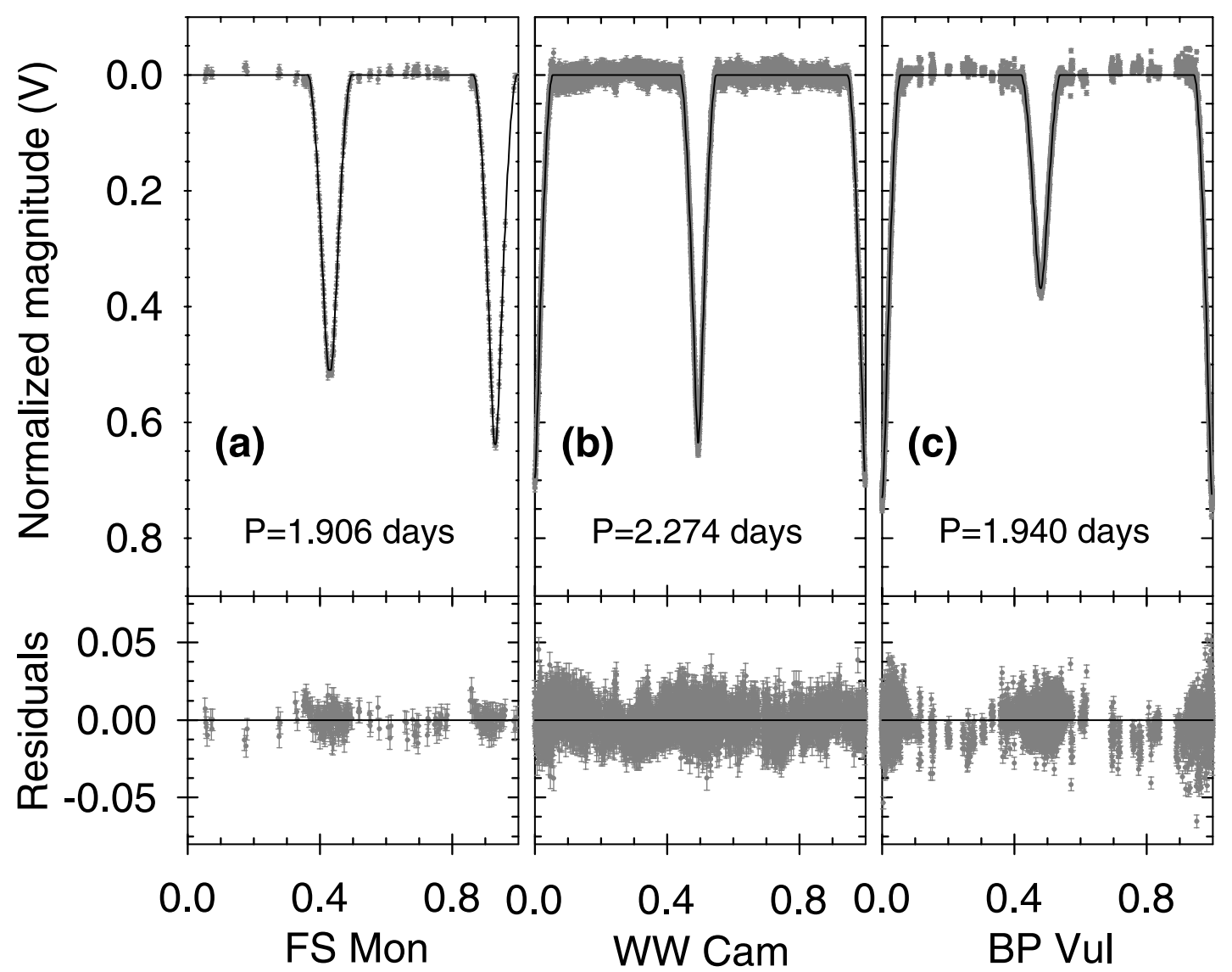

FIG. 3.- Observed light curves of FS Mon (Lacy et al. 2000), WW Cam (Lacy et al. 2002), and BP Vul (Lacy et al. 2003), each overplotted with the best-fit model DEBiL solution used in our MECI algorithm. The masses and ages corresponding to these solutions are listed in Table 1. The residuals to each fit are shown in the lower panels.

the data and the errors are Gaussian-distributed and are estimated correctly.

In practice, typical light curves may have $N_{\text {lc }}>1000$ points, whereas only $1 \leq N_{\text {colors }} \leq 5$ might be available. We have found it necessary to select a value for $w$ that increases the relative weight of the color information to obtain reliable results $\left(w<N_{\mathrm{lc}}\right)$. In general, the optimal value for $w$ will depend on the accuracy of the observed colors $\tilde{O}_{c}$ and the degree to which the EB light curve deviates from the assumption of two well-detached, limbdarkened spherical components. Based on the tests described in $\S 4$, we find that a wide range of values for $w$ produces similar results, and that values in the range $10 \leq w \leq 100$ most accurately recover the correct values for the stellar parameters.

We identify the global minimum of $\chi_{\nu}^{2}$ in three steps. First, we calculate the value of $\chi_{\nu}^{2}$ at all points in a coarse $N \times N$ grid at each age slice. The $N$ mass values are selected to be spaced from the lowest mass value present in the models to the greatest values at which the star has not yet evolved off the main sequence. Next, we identify any local minima, and refine their values by evaluating all available intermediate-mass pairings. Finally, we identify the global minimum from the previous step, and fit an elliptic paraboloid to the local $\chi_{\nu}^{2}$ surface around the lowest minimum. We assign the most likely values for the stellar masses and age to be the location of the minimum of the paraboloid. The curvature of the paraboloid in each axis provides the estimates of the uncertainties in these parameters. In practice, these formal uncertainties underestimate the true uncertainties since they do not consider the systematic errors due to (1) the oversimplified EB model, (2) errors in the theoretical stellar isochrones and limb-darkening coefficients, and (3) sources of non-Gaussian noise in the data.

When choosing the value of $N$ above, we must balance computational speed considerations with the risk of missing the global minimum by undersampling the $\chi_{\nu}^{2}$ surface. For most mainsequence EBs, the $\chi_{\nu}^{2}$ surface contains only one, or at most a few local minima, and our experience is that $N=10$ usually suffices (see $\S 4.2$ ). For systems that are either very young or in which a component has begun to evolve off the main sequence, the $\chi_{\nu}^{2}$ surface requires a much denser sampling. Evolved components, which may be present in as many as a third of the EBs of a magnitude-limited photometric survey (Alcock et al. 1997), introduce an additional challenge if their isochrones intersect other isochrones on the color-magnitude diagram. At such intersection points, stars of different masses will have approximately equal sizes and effective temperatures, creating degenerate regions on the $\chi_{\nu}^{2}$ surface. This degeneracy can, in principal, be broken with sufficient color information, which will probe differences in the stars' limb darkening and absorption features, both of which vary with surface gravity.

We also note that multiple local minimum may result for light curves with very small formal uncertainties. In this case, numerical errors in the simulated light curve dominate. This problem can be mitigated by increasing the number of iterations used in fitting the orbital parameters (see $\S 3.2$ ).

\subsection{Optimization}

We implemented a number of optimizations to increase the speed of MECI. First, since each light curve is independent, we 


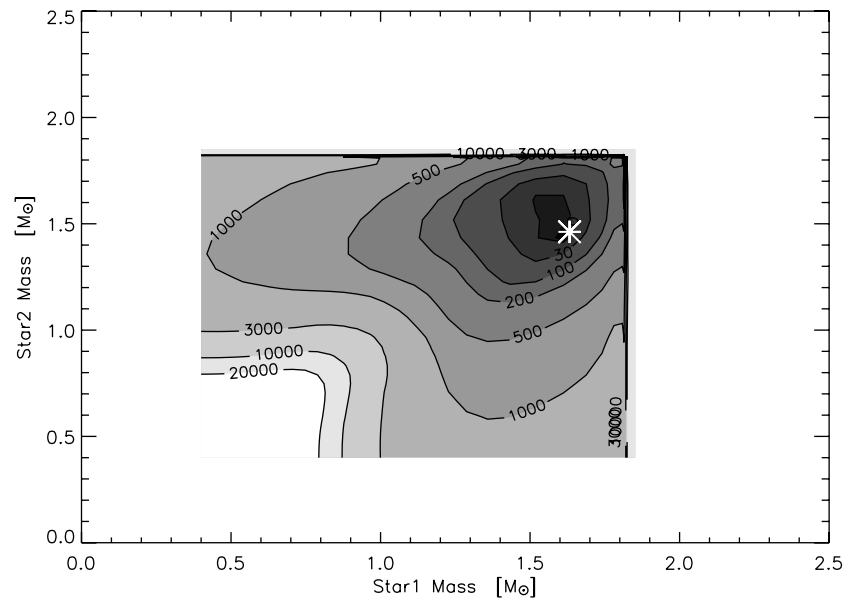

FIG. 4.- MECI $\chi_{\nu}^{2}$ surface to the FS Mon light curve and colors (Lacy et al. 2000 ), assuming an age of $1.6 \mathrm{Gyr}$ and fixing $w=10$. The estimate of the stellar masses (Lacy et al. 2000) from a combined analysis of the light curve and spectroscopic observations is indicated by a white asterisk and is near to the minimum identified by MECI. Note the erratic behavior of the contours at the upper end of the mass range, which results from the rapid evolution of stars of those masses at this age.

parsed the data set and ran MECI in parallel on multiple CPUs. Second, we reduced the number of operations by identifying and skipping unphysical stellar pairings. Specifically, we required $\left(r_{1}+r_{2}<0.8\right)$ to preclude binaries that were not well detached. In addition, for EBs with clear primary and secondary eclipses, we skipped high-contrast-ratio pairings for which the maximum depth of the primary eclipse, $\Delta m_{1}$, or the maximum depth of the secondary eclipse, $\Delta m_{2}$, fell below a specified threshold, $\Delta m_{\text {cutoff }}$. In particular, we skipped over pairings for which $\min \left(\Delta m_{1}, \Delta m_{2}\right) \leq \Delta m_{\text {cutoff }}$, where

$$
\begin{aligned}
& \Delta m_{1} \simeq 2.5 \log \left[1+\frac{\left(R_{2} / R_{1}\right)^{2}}{\left.1-\left(R_{2} / R_{1}\right)^{2}+10^{0.4\left(M_{1}-M_{2}\right)}\right]}\right. \\
& \Delta m_{2}=2.5 \log \left[1+10^{0.4\left(M_{1}-M_{2}\right)}\right] .
\end{aligned}
$$

These estimates assume equatorial eclipses, since we seek to evaluate the maximum possible eclipse depths. The first expression is approximate because it neglects the effects of limbdarkening on the eclipse depth. In practice, the chosen value for $\Delta m_{\text {cutoff }}$ will depend on the typical precision and cadence of the data set in question.

We note here a special case that we revisit in $\S 4.3$. For EB light curves with equally spaced eclipses of equal depth, we must also consider the possibility that our assumed period is double the true value, and hence the secondary eclipse is undetected. When we identified such cases, we analyzed the light curve as usual but removed the above requirement. In such cases, we can place only an upper limit on the mass of the secondary component.

\section{TESTING MECI}

In order to establish the accuracy and reliability of MECI under a variety of scenarios, we conducted two distinct tests.

\subsection{Observed Systems}

The first test was to run MECI on several observed light curves of eclipsing binary systems whose stellar parameters had been precisely determined from detailed photometric and spectroscopic

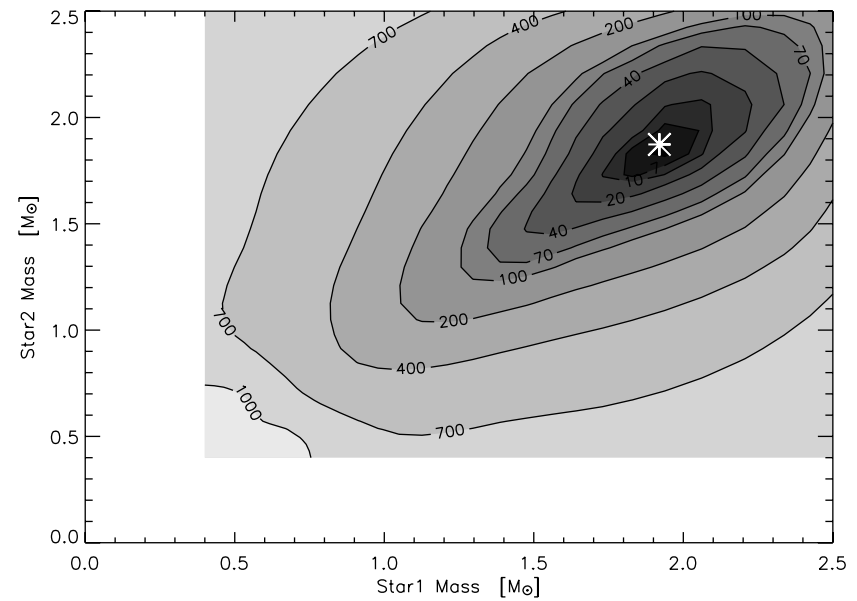

FIG. 5.- MECI $\chi_{\nu}^{2}$ surface to the WW Cam light curve and colors (Lacy et al. 2002), assuming an age of $0.6 \mathrm{Gyr}$ and fixing $w=10$. The estimate of the stellar masses (Lacy et al. 2002) from a combined analysis of the light curve and spectroscopic observations is indicated by a white asterisk and is extremely close to the solution identified by MECI.

studies. We examined three well-studied EBs. The first was FS Monocerotis (Lacy et al. 2000), for which we modeled the published light curve, which had $N_{\text {lc }}=249$ data points, as well as the published $U-B$ and $B-V$ colors. The second was WW Camelopardalis (Lacy et al. 2002), for which we modeled the published light curve, which had $N_{\mathrm{lc}}=5759$ observations, as well as the $B-V$ color. Finally, we studied BP Vulpeculae (Lacy et al. 2003), for which we modeled the published light curve, which had $N_{\text {lc }}=5236$ observations, as well as the $B-V$ color. All three published light curves were observed in $V$ band and are plotted in Figure 3. The colors had been corrected for reddening. The contour plots of the $\chi_{\nu}^{2}$ surfaces resulting from our MECI analysis (setting the weighting $w=10$ ) are shown in Figures 4, 5, and 6. Note that FS Mon is more tightly constrained due to its greater color information. Furthermore, the asymmetry in BP Vol's contour is due to its unequal eclipse depths. In all cases, the $\chi_{\nu}^{2}$ surface has a single minimum, which is close to the published values. In Table 1, we tabulate the results of our analysis and compare these to the published values.

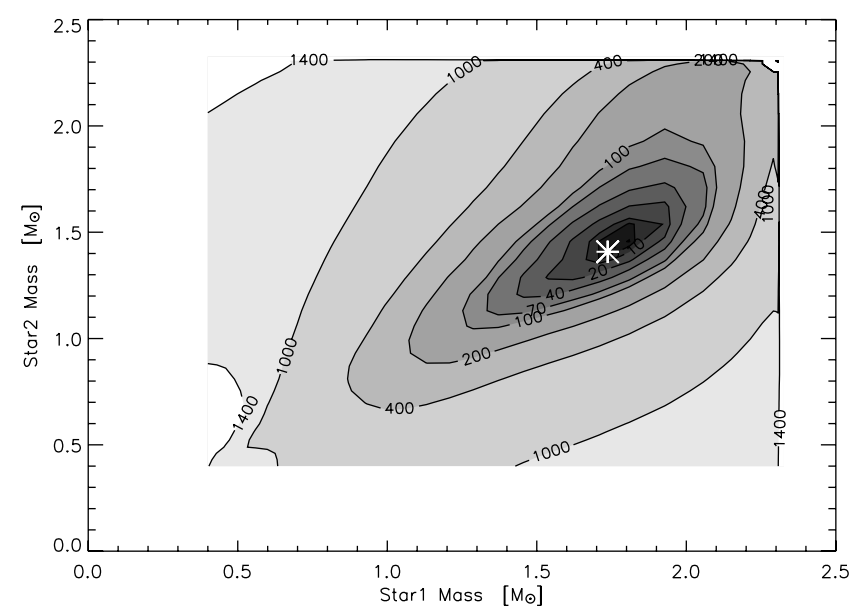

FIG. 6.-MECI $\chi_{\nu}^{2}$ surface to the BP Vul light curve and colors (Lacy et al. 2003 ), assuming an age of 0.8 Gyr and fixing $w=10$. The estimate of the stellar masses (Lacy et al. 2003) from a combined analysis of the light curve and spectroscopic observations is indicated by a white asterisk and is extremely close to the solution identified by MECI. 
TABLE 1

Accuracy of MECi Parameter Estimates for Three Well-Studied Binaries

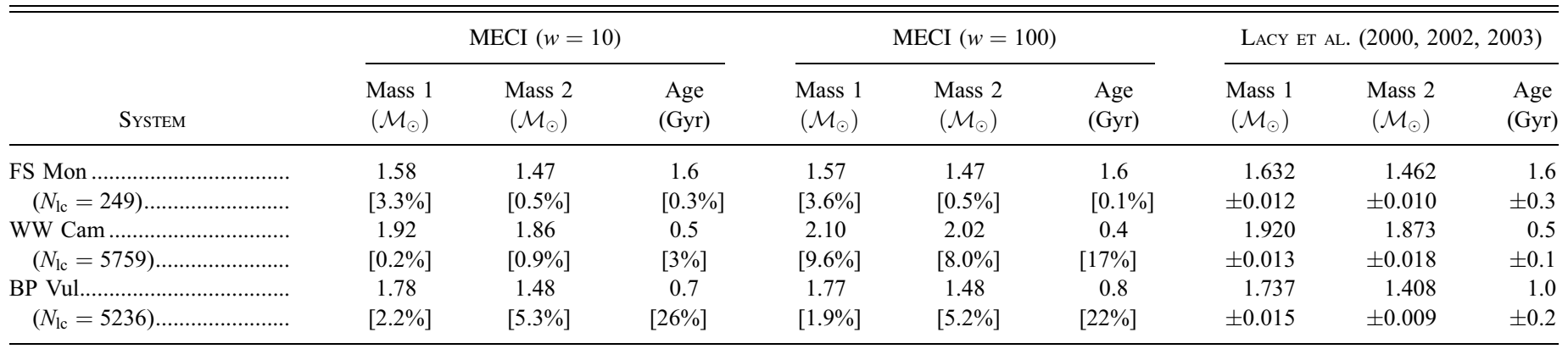

Notes.-The rightmost columns list the masses, ages, and errors of the component stars as determined by a combined analysis of their light curves and spectroscopic orbits (Lacy et al. 2000, 2002, 2003). The leftmost columns list the estimates of these quantities produced by MECI assuming $w=10$, and the central columns list the estimates from MECI assuming $w=100$. The square brackets indicate the fractional errors of the MECI results with respect to the numbers in the rightmost columns.

We then changed the weighting factor to $w=100$ and repeated this procedure. The MECI results for FS Mon and BP Vul were essentially identical to our earlier findings for $w=10$. In the case of WW Cam, the results for $w=10$ were significantly closer to the published values. This is likely due to the fact that it is a young system (age $=500 \mathrm{Myr}$ ), for which the brightness and radii at constant mass vary significantly. Thus, the lower lightcurve information weighting brought about smoother $\chi_{\nu}^{2}$ contours (see $\S 3.3$ ).

\subsection{Simulated Systems}

In our second test, we produced large numbers of simulated EB light curves with various levels of injected noise, and subsequently analyzed these photometric data sets with MECI. We then compared the input and derived estimates of the stellar masses and ages in order to quantify the accuracy of the MECI analysis.

We selected the orbital and stellar parameters of each simulated EB as follows. First, we drew an age at random from a uniform probability distribution between $200 \mathrm{Myr}$ and $10 \mathrm{Gyr}$. We then selected the masses of the two EB components independently from a flat distribution from $0.4 \mathcal{M}_{\odot}$ and the maximum mass at which stars of this age would still be located on the main sequence. We then assigned the orbital period by drawing a number from a uniform probability distribution spanning $0<P \leq 10$ days. Similarly, we assigned the epoch of perihelion by drawing from a uniform probability distribution spanning $0 \leq t_{0}<P$ and the orbital inclination from a uniform distribution within the range that produces eclipses, $\arccos \left(r_{1}+r_{2}\right) \leq i \leq \pi / 2$. For the tests of eccentric systems, we also randomly selected an eccentricity, uni- formly from $0 \leq e \leq 0.1$, and randomly selected the angle of perihelion, uniformly from $0 \leq \omega<2 \pi$. Finally, we rejected any EB system if its components were overlapping or in near contact, $r_{1}+r_{2} \geq 0.8$. We also filtered out EBs with undersampled eclipses, or for which one of the eclipse depths was smaller than the assumed $1 \sigma$ noise level.

Each simulated light curve contained $1000 R$-band data points, to which we injected Gaussian-distributed noise. When color information was required, we computed the out-of-eclipse photometric colors for each EB, and injected a 0.02 mag Gaussian-distributed error to this value. The colors we considered were $(V-I)_{\mathrm{C}}$, which is similar to the color provided by the OGLE II catalog (Wozniak et al. 2002), as well as $(J-H)_{\mathrm{ESO}}$ and $(H-K)_{\mathrm{ESO}}$, which are similar to the colors provided by the 2MASS catalog (Kleinmann et al. 1994). ${ }^{5}$

We simulated 8 sets of 2500 systems each, with the sets differing in the following respects (see Table 2): (1) circular or eccentric orbits, (2) the number of points in the search grid, (3) the value of $w$, which describes the relative weight between the color and photometric data, and (4) the availability of color information.

In order to summarize the accuracy of the MECI results, we computed the quadrature sum of the relative differences between the assumed and derived values for the masses of the two components. We plot the histograms of these values in Figure 7. In each histogram, we identify the value encompassing the region

\footnotetext{
5 The 2MASS catalog uses custom $J, H$, and $K_{s}$ filters, which can be approximately converted to the ESO standard using linear transformations (Carpenter 2001).
}

TABLE 2

Accuracy of MECi Mass Estimates for Simulated Systems

\begin{tabular}{|c|c|c|c|c|c|c|}
\hline Set & $\begin{array}{l}\text { Noise } \\
(\mathrm{mag})\end{array}$ & Orbit & Search Grid & Weighting & Color Information & $\begin{array}{c}\text { 90th Percentile Error } \\
(\%)\end{array}$ \\
\hline $\mathrm{A}$ & 0.01 & circular & $15 \times 15$ & N/A & No color information & 30 \\
\hline 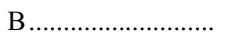 & 0.01 & circular & $15 \times 15$ & $w=100$ & $(V-I)_{\text {Cousins }}$ & 5.9 \\
\hline $\mathrm{C}$ & 0.01 & circular & $15 \times 15$ & $w=100$ & $(J-H)_{\mathrm{ESO}}$ and $(H-K)_{\mathrm{ESO}}$ & 5.8 \\
\hline $\mathrm{D}$ & 0.001 & circular & $15 \times 15$ & $w=100$ & $(J-H)_{\mathrm{ESO}}$ and $(H-K)_{\mathrm{ESO}}$ & 4.0 \\
\hline $\mathrm{E}$ & 0.01 & circular & $15 \times 15$ & $w=10$ & $(J-H)_{\mathrm{ESO}}$ and $(H-K)_{\mathrm{ESO}}$ & 6.6 \\
\hline $\mathrm{F}$ & 0.01 & circular & $10 \times 10$ & $w=100$ & $(J-H)_{\mathrm{ESO}}$ and $(H-K)_{\mathrm{ESO}}$ & 6.1 \\
\hline $\mathrm{G}$ & 0.01 & eccentric & $15 \times 15$ & $w=100$ & $(J-H)_{\mathrm{ESO}}$ and $(H-K)_{\mathrm{ESO}}$ & 8.8 \\
\hline $\mathrm{H}$ & 0.001 & eccentric & $15 \times 15$ & $w=100$ & $(J-H)_{\mathrm{ESO}}$ and $(H-K)_{\mathrm{ESO}}$ & 23 \\
\hline
\end{tabular}

Notes.-The parameters of the 8 distinct sets of simulated EB light curves that we generated and subsequently analyzed with MECI. The rightmost column lists the range of the quadrature sum of the fractional errors on the masses, which encompasses $90 \%$ of the solutions (see Fig. 7), which we take to be indicative of the accuracy of MECI under the specified conditions. 

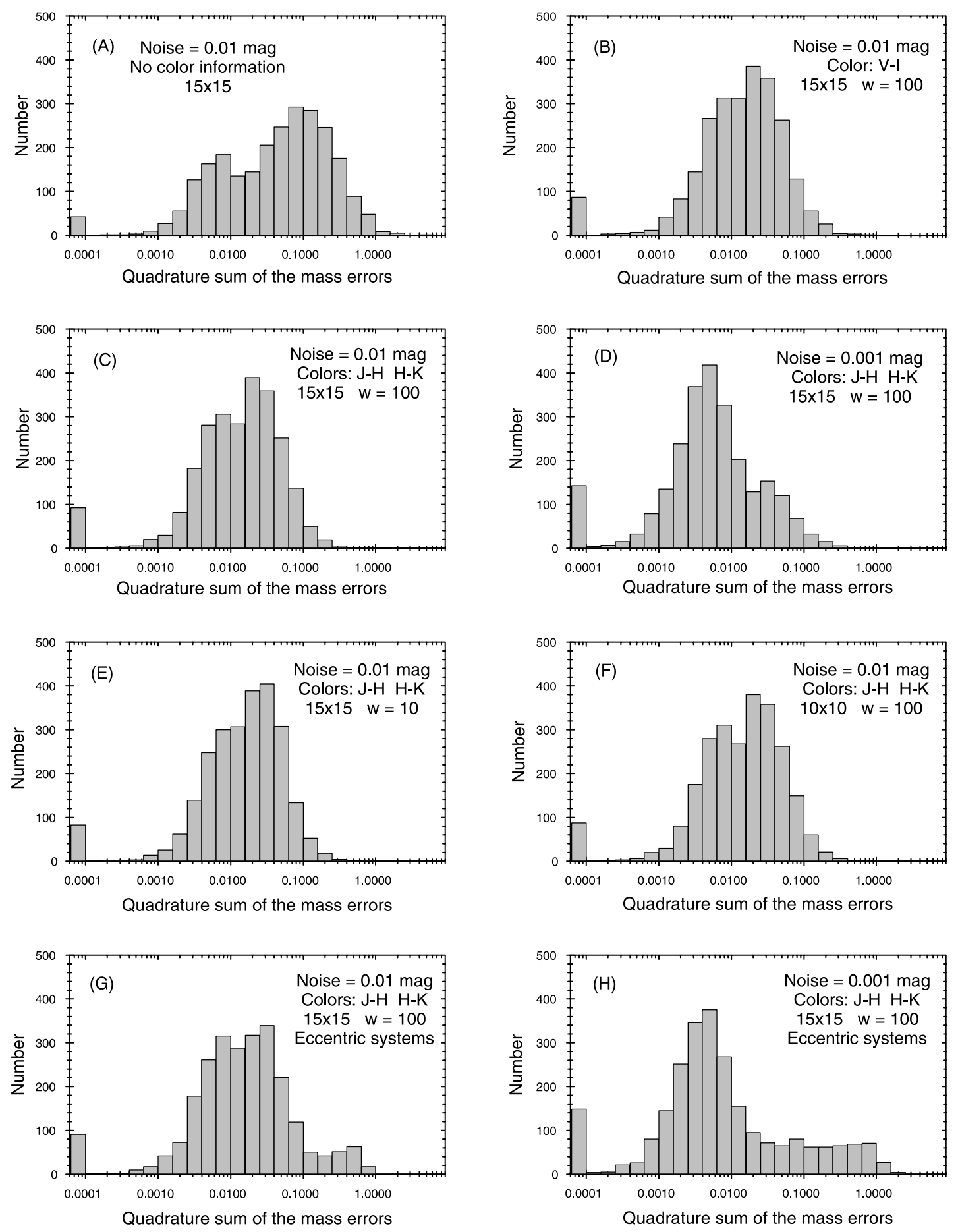

Fig. 7.- Histograms of the quadrature sums of the relative differences in the assumed and calculated masses for the stellar components for each of the sets of simulated light curves described in Table 2. Each set contains 2500 simulated EBs as described in $\S 4.2$, and the key parameters of each set are listed in the upper right corner of each panel. The leftmost bin contains the sum of all results with values less than 0.0001 . The ability of the method to accurately assign the masses to the component stars degrades significantly in the absence of any color information (upper left panel), but is generally robust against changes in the particular choice of $w$ or $N($ see $\S 4.2)$.

that contains $90 \%$ of the results. We call this range the " 90 th percentile error," and list it in the final column of Table 2.

We find that the inclusion of color information significantly improves the accuracy of the MECI results, lowering the 90th percentile error from $30 \%$ in set $A$ to less than $6 \%$ in sets $\mathrm{B}$ and C. In contrast, changing the value of $w$ from 100 in set $\mathrm{C}$ to 10 in set $E$ results in only a modest increase of $0.8 \%$ in the size of the 90th percentile error. This indicates that the results are robust to the particular choice of $w$. We note, however, that a value of $w>100$ will usually provide too little weight to the color information, which results in poorer accuracy. An extreme example of this is seen in set A.

Similarly, MECI is not sensitive to the exact value of the search grid size. In particular, decreasing the grid size from $15 \times$ 15 in set $\mathrm{C}$ to $10 \times 10$ in set $\mathrm{F}$ increases the 90th percentile error only modestly, from $5.8 \%$ to $6.1 \%$. This stability results from the 
fact that the $\chi_{\nu}^{2}$ function contains a broad minimum, which is well sampled even with $N=10$ grid points. We note, however, that this is no longer the case when considering evolved star systems (e.g., $\S 3.3$ ), for which a larger number of grid points is required.

When we decreased the level of the noise injected into the photometric time series from $0.01 \mathrm{mag}$ in set $\mathrm{C}$ to $0.001 \mathrm{mag}$ in set $\mathrm{D}$, the 90 th percentile error dropped from $5.8 \%$ to $4.0 \%$. Surprisingly, the tail of the upper end of the error distribution extends to larger values in set $\mathrm{D}$. This appears to be due to the phenomenon discussed in $\S 3.3$, whereby the $\chi_{\nu}^{2}$ function occasionally contains many local minima. This problem becomes acute for eccentric systems, since they have a far more complex $\chi_{\nu}^{2}$ function. Decreasing their noise from $0.01 \mathrm{mag}$ in set $\mathrm{G}$ to $0.001 \mathrm{mag}$ in set $\mathrm{H}$ raises the 90th percentile error from $8.8 \%$ to $23 \%$. This relatively poor performance reflects the algorithm's inability to robustly identify the global minimum under these conditions. In such cases one must increase the size of the search grid and iteratively solve for the orbital parameters of the systems, which results in a significant increase in the computational time.

\subsection{Limitations}

A significant degeneracy results for light curves in which two distinct eclipses are not apparent. For such systems, two distinct possibilities exist, namely that either the EB consists of two twin components with an orbital period $P$, or that the EB consists of two stars with very disparate sizes (such that the secondary eclipse is not discernible), with an orbital period $2 P$. It is often necessary to flag such systems and conduct analyses with both possible values for the orbital periods. Distinguishing which of these possibilities is the correct solution is challenging, but in some instances there are clues. One such clue is a variable light-curve plateau that results from the mutual tidal distortions, which in turn might indicate the true orbital period (twice that of the observed modulation). A second possibility is a red excess in the system color indicating a low-mass secondary. Of course, follow-up spectroscopic observations can readily resolve this degeneracy, either by indicating the presence of two components of similar brightness, or through a direct determination of the orbital period.
We note that MECI employs a simplified model for the generation of the light curves (DEBiL), which can bring about additional complications when applied to systems in which our assumptions (see $\S 3.2$ ) do not hold. For example, our model ignores the effect of third light, from either a physically associated star or a chance superposition, which reduces the apparent depths of the eclipses and may contaminate the estimate of the system color. Furthermore, we have ignored reflection effects, which can raise the light-curve plateau at times immediately preceding or following eclipses. Finally, tidal distortions will increase the apparent system brightness at orbital quadrature, which can serve to increase the apparent depth of the eclipses. In order for MECI to be able to properly handle these cases, its light-curve generator must be replace with a more sophisticated one (e.g., WD or EBOP), which will likely make MECI significantly more computationally expensive.

\section{CONCLUSIONS}

We have described a method for identifying an EB's components using only its photometric light curve and combined colors. By utilizing theoretical isochrones and limb-darkening coefficients, this method greatly reduces the EB parameter space over which one needs to search. Using this approach, we can quickly estimate the masses, radii, and absolute magnitudes of the components, without spectroscopic data. We described an implementation of this method, which enables the systematic analyses of data sets consisting of photometric time series of large numbers of stars, such as those produced by OGLE, MACHO, TrES, HAT, and many others. Such techniques are expected to grow in importance with the next generation surveys, such as Pan-STARRS (Kaiser et al. 2002) and LSST (Tyson 2002). In a future publication, we shall describe a specific application of these codes, namely to search for low-mass eclipsing binaries in the TrES data set.

We would like to thank Guillermo Torres for many useful discussions and critiques, and we would like to thank Tsevi Mazeh for sharing his ideas regarding applications for this method. We would also like to thank Sarah Dykstra for her help editing this paper.
Alard, C. 2000, A\&AS, 144, 363

Alard, C., \& Lupton, R. H. 1998, ApJ, 503, 325

Alcock, C., et al. 1997, AJ, 114, 326

1998, ApJ, 492, 190

Alonso, R., et al. 2004, ApJ, 613, L153

Andersen, J. 1998, in IAU Symp. 189, Fundamental Stellar Properties, ed. T. R. Bedding, A. J. Booth, \& J. Davis (Dordrecht: Kluwer), 99

Bakos, G., Noyes, R. W., Kovács, G., Stanek, K. Z., Sasselov, D. D., \& Domsa, I. 2004, PASP, 116, 266

Baraffe, I., Chabrier, G., Allard, F., \& Hauschildt, P. H. 1998, A\&A, 337, 403 Carpenter, J. M. 2001, AJ, 121, 2851

Charbonneau, D., Brown, T. M., Burrows, A., \& Laughlin, G. 2006, in Protostars and Planets V, ed. B. Reipurth, D. Jewitt, \& K. Keil (Tucson: Univ. Arizona Press), in press (astro-ph/0603376)

Claret, A. 1998, A\&A, 335, 647

. 2000, A\&A, 363, 1081

Claret, A., Diaz-Cordoves, J., \& Gimenez, A. 1995, A\&AS, 114, 247

Creevey, O. L., et al. 2005, ApJ, 625, L127

Crotts, A. P. S. 1992, ApJ, 399, L43

Delfosse, X., Forveille, T., Mayor, M., Burnet, M., \& Perrier, C. 1999, A\&A, 341, L63

Devor, J. 2004, BAAS, 205, 1813

. 2005, ApJ, 628, 411

Devor, J., \& Charbonneau, D. 2006, preprint (astro-ph/0510067)

Duquennoy, A., \& Mayor, M. 1991, A\&A, 248, 485

\section{REFERENCES}

Etzel, P. B. 1981, in Photometric and Spectroscopic Binary Systems, ed. E. B. Carling \& Z. Kopal (NATO ASI Ser. C., 69; Dordrecht: Reidel), 111 1991, IAPPP, 45, 25

Guinan, E. F., Bradstreet, D. H., \& Dewarf, L. E. 1996, ASP Conf. Ser. 90 The Origins, Evolution, and Destinies of Binary Stars in Clusters, ed. E. F. Milone \& J.-C. Mermilliod (San Francisco: ASP), 196

Kaiser, N., et al. 2002, Proc. SPIE, 4836, 154

Kallrath, J., \& Milone, E. F. 1999, Eclipsing Binary Stars: Modeling and Analysis (New York: Springer)

Kim, Y., Demarque, P., Yi, S. K., \& Alexander, D. R. 2002, ApJS, 143, 499

Kleinmann, S. G., et al. 1994, Ap\&SS, 217, 11

Kopal, Z. 1959, Close Binary Systems (London: Chapman \& Hall)

Kron, G. E. 1952, ApJ, 115, 301

Kurucz, R. L. 1992, in IAU Symp. 149, The Stellar Populations of Galaxies, ed. B. Barbuy \& A. Renzini (Dordrecht: Kluwer), 225

Lacy, C. H. 1977a, ApJS, 34, 479 1977b, ApJ, 218, 444

Lacy, C. H. S., Torres, G., Claret, A., \& Sabby, J. A. 2002, AJ, 123, 1013 2003, AJ, 126, 1905

Lacy, C. H. S., Torres, G., Claret, A., Stefanik, R. P., Latham, D. W., \& Sabby, J. A. 2000, AJ, 119, 1389

Latham, D. W. 1992, in IAU Colloq. 135, Complementary Approaches to Double and Multiple Star Research, ed. H. A. McAlister \& W. I. Hartkopf (ASP Conf. Ser. 32; Chelsea: ASP), 110

Maceroni, C., \& Montalbán, J. 2004, A\&A, 426, 577 
Maceroni, C., \& Rucinski, S. M. 1997, PASP, 109, 782

Mazeh, T., Tamuz, O., \& North, P. 2006, in Close Binaries in the 21st Century: New Opportunities and Challenges, ed. A. Gimenez, E. Guinan, P. Niarchos, \& S. Rucinski, in press

McCullough, P. R., et al. 2006, ApJ, 648, 1228

Metcalfe, T. S., Mathieu, R. D., Latham, D. W., \& Torres, G. 1996, ApJ, 456, 356

Nelder, J. A., \& Mead, R. 1965, Computer J., 7, 308

O’Donovan, F. T., et al. 2006, ApJ, 644, 1237

Paczynski, B. 1997, in Proc. STScI Symp. (1996 May), The Extragalactic Distance Scale, ed. M. Livio, M. Donahue, \& N. Panagia (Baltimore: STScI), 273

Popper, D. M., \& Etzel, P. B. 1981, AJ, 86, 102

Pourbaix, D., et al. 2004, A\&A, 424, 727

Press, W. H., Teukolsky, S. A., Vetterling, W. T., \& Flannery, B. P. 1992, Numerical Recipes in C: The Art of Scientific Computing (2nd ed.; Cambridge: Univ. Press)
Ribas, I. 2003, A\&A, 398, 239

Schwarzenberg-Czerny, A. 1989, MNRAS, 241, 153

1996, ApJ, 460, L107

Stebbing, J. 1910, ApJ, 32, 185

Torres, G., \& Ribas, I. 2002, ApJ, 567, 1140

Tyson, J. A. 2002, Proc. SPIE, 4836, 10

Udalski, A., et al. 1994, Acta Astron., 44, 165

Vallée, J. P. 2005, AJ, 130, 569

Wambsganss, J. 2006, in Proc. of the 33rd Saas-Fee Advanced Course, Gravitational Lensing: Strong, Weak and Micro, ed. G. Meylan, P. Jetzer, \& P. North (Berlin: Springer), 457

Wilson, R. E., \& Devinney, E. J. 1971, ApJ, 166, 605

Wozniak, P. R. 2000, Acta Astron., 50, 421

Wozniak, P. R., Udalski, A., Szymanski, M., Kubiak, M., Pietrzynski, G.,

Soszynski, I., \& Zebrun, K. 2002, Acta Astron., 52, 129

Wyithe, J. S. B., \& Wilson, R. E. 2001, ApJ, 559, 260 2002, ApJ, 571, 293 